\title{
Oxidative stress in relation to diet and physical activity among premenopausal women
}

\author{
Chelsea Anderson ${ }^{1}$, Ginger L. Milne ${ }^{2}$, Dale P. Sandler ${ }^{3}$ and Hazel B. Nichols ${ }^{1 *}$ \\ ${ }^{1}$ Department of Epidemiology, University of North Carolina Gillings School of Global Public Health, Chapel Hill, NC 27599, USA \\ ${ }^{2}$ Division of Clinical Pharmacology, Vanderbilt University Medical Center, Nashville, TN 37232, USA \\ ${ }^{3}$ Epidemiology Branch, National Institute of Environmental Health Sciences, National Institutes of Health, Durham, NC 27709, USA
}

(Submitted 18 May 2016 - Final revision received 27 July 2016 - Accepted 5 August 2016 - First published online 11 October 2016$)$

\section{Abstract}

Higher levels of oxidative stress, as measured by $\mathrm{F}_{2}$-isoprostanes, have been associated with chronic diseases such as CVD and some cancers. Improvements in diet and physical activity may help reduce oxidative stress; however, previous studies regarding associations between lifestyle factors and $\mathrm{F}_{2}$-isoprostane concentrations have been inconsistent. The aim of this cross-sectional study was to investigate whether physical activity and intakes of fruits/vegetables, antioxidant nutrients, dietary fat subgroups and alcohol are associated with concentrations of $\mathrm{F}_{2}$-isoprostane and the major $\mathrm{F}_{2}$-isoprostane metabolite. Urinary $\mathrm{F}_{2}$-isoprostane and its metabolite were measured in urine samples collected at enrolment from 912 premenopausal women (aged 35-54 years) participating in the Sister Study. Physical activity, alcohol consumption and dietary intakes were self-reported via questionnaires. With adjustment for potential confounders, the geometric means of $\mathrm{F}_{2}$-isoprostane and its metabolite were calculated according to quartiles of dietary intakes, alcohol consumption and physical activity, and linear regression models were used to evaluate trends. Significant inverse associations were found between $\mathrm{F}_{2}$-isoprostane and/or its metabolite and physical activity, vegetables, fruits, vitamin $\mathrm{C}, \alpha$-carotene, vitamin E, $\beta$-carotene, vitamin $\mathrm{A}$, Se, lutein + zeaxanthin and long-chain $n$ - 3 fatty acids. Although trans fats were positively associated with both $\mathrm{F}_{2}$-isoprostane and its metabolite, other dietary fat subgroups including SFA, $n$ - 6 fatty acids, $n$ - 3 fatty acids, MUFA, PUFA, short-chain $n-3$ fatty acids, long-chain $n-3$ fatty acids and total fat were not associated with either $\mathrm{F}_{2}$-isoprostane or its metabolite. Our findings suggest that lower intake of antioxidant nutrients and higher intake of trans fats may be associated with greater oxidative stress among premenopausal women.

Key words: Oxidative stress: F $_{2}$-isoprostanes: Diet: Physical activity: Premenopausal women

Oxidative stress, an unfavourable imbalance between production of reactive oxygen species and antioxidant defence, has been implicated in the aetiology of several chronic diseases including CVD, diabetes and some cancers ${ }^{(1-5)}$. Although some chronic disease risk factors such as smoking have been consistently associated with higher levels of oxidative stress ${ }^{(6,7)}$, relationships with other lifestyle factors such as diet, alcohol consumption and physical activity have not been well characterised in healthy adults.

Diet may be linked to oxidative stress through the consumption of antioxidants - substances that inhibit the oxidation of body substrates by reactive oxygen species. Nutrients with established antioxidant activity include carotenoids ( $\beta$-carotene, $\alpha$-carotene, lycopene, cryptoxanthin, lutein and zeaxanthin), vitamin C (ascorbic acid), vitamin E ( $\alpha$-tocopherol), Se and $\mathrm{Zn}^{(8)}$. While some of these nutrients such as carotenoids act directly by quenching singlet molecular oxygen and free radicals $^{(9,10)}$, others such as $\mathrm{Zn}$ act indirectly as cofactors of antioxidant enzymes ${ }^{(11)}$. Although some studies have observed lower oxidative stress levels with higher dietary intakes of various antioxidant nutrients, such associations have not been demonstrated consistently ${ }^{(12-17)}$. Similarly, consumption of fruits and vegetables, foods rich in antioxidants, has been inversely associated with oxidative stress in some, but not all, observational studies ${ }^{(12,18-20)}$.

Dietary fats may also be related to oxidative stress levels. Though n-3 fatty acids have been associated with lower oxidative stress in some reports ${ }^{(21,22)}$, higher intakes of trans fat and SFA were related to higher oxidative stress in a recent study among midlife women ${ }^{(13)}$. However, human studies investigating the relationships between various dietary fat subgroups and oxidative stress are limited.

Associations between oxidative stress and other behaviours such as physical activity and alcohol consumption are also uncertain. While acute, vigorous exercise appears to increase oxidative stress, chronic, moderate-intensity physical activity

Abbreviations: 15- $\mathrm{F}_{2 \mathrm{t}}$-IsoP-M, 2,3-dinor-5,6-dihydro-15- $\mathrm{F}_{2 \mathrm{t}}$-isoprostane; $\mathrm{F}_{2}$-IsoP, $\mathrm{F}_{2}$-isoprostanes.

* Corresponding author: H. B. Nichols, email hazel.nichols@unc.edu 
may have the opposite effect over the long term ${ }^{(23,24)}$. Similarly, excess consumption of alcohol is linked to oxidative stress through ethanol metabolism, which involves the production of reactive oxygen species ${ }^{(25)}$. However, the effect of regularly consuming moderate amounts of alcohol remains unclear.

Although numerous biomarkers of oxidative stress exist, $\mathrm{F}_{2}$-isoprostanes $\left(\mathrm{F}_{2}\right.$-IsoP), generated from free radical-catalysed peroxidation of arachidonic acid, are considered to be among the most accurate ${ }^{(26,27)} \cdot F_{2}$-IsoP have been positively associated with chronic disease risk factors such as obesity and smoking ${ }^{(28)}$. Though prospective studies to date are limited, some evidence has linked elevated $\mathrm{F}_{2}$-IsoP to risk of CVD and certain cancers $^{(1-3,5)}$. Urinary $\mathrm{F}_{2}$-IsoP are a particularly stable biomarker of oxidative stress, as they are not subject to autoxidation during sample collection and storage, unlike blood plasma measures ${ }^{(29)}$. Furthermore, while local renal production may affect the excretion of unmetabolized $\mathrm{F}_{2}$-IsoP in human urine, the metabolised form of $15-\mathrm{F}_{2 \mathrm{t}}$-isoprostane - 2,3-dinor-5,6dihydro- $15-\mathrm{F}_{2 \mathrm{t}}$-isoprostane $\left(15-\mathrm{F}_{2 \mathrm{t}}-\mathrm{IsoP}-\mathrm{M}\right)$ - is unaffected by renal production ${ }^{(30)}$. However, most previous studies on $\mathrm{F}_{2^{-}}$ isoprostanes and lifestyle factors have relied exclusively on unmetabolized $\mathrm{F}_{2}$-IsoP. Therefore, the purpose of this crosssectional study was to evaluate associations of both $\mathrm{F}_{2}$-IsoP and $15-\mathrm{F}_{2 \mathrm{t}}$-IsoP-M with physical activity, alcohol consumption, and intakes of dietary fats, fruits and vegetables, and antioxidant nutrients among healthy, premenopausal women.

\section{Methods}

Participants included in this analysis were controls of a case-control study on incident breast cancer nested within the Sister Study - a prospective observational cohort of US women designed to identify risk factors for breast cancer. The objective of the nested case-control study was to evaluate novel biomarkers of premenopausal breast cancer risk. Women aged 35-74 years from the USA and Puerto Rico were recruited for the Sister Study from 2003 to 2009 through a national advertising campaign and a network of breast cancer professionals and recruitment volunteers. All of them had a sister who had been diagnosed with breast cancer, but were themselves free of breast cancer at enrolment. All participants provided their informed written consent. The study was approved by the Institutional Review Board of the National Institute of Environmental Health Sciences, the National Institutes of Health and the Copernicus Group.

\section{Population for analysis}

Women were eligible to be included in the control sample if they were aged 35-54 years, premenopausal, had at least one intact ovary and had a urine sample collected at enrolment. Those who reported one or more menstrual cycles in the previous 12 months were categorised as premenopausal, as were women aged 54 years and younger whose only reason for not experiencing menses was hysterectomy (without bilateral oophorectomy). A total of 912 women, who did not have a breast cancer diagnosis as of 31 December 2012, had urine samples analysed for $\mathrm{F}_{2}$-isoprostanes and were eligible for this analysis.

\section{Measurement of $F_{2}$-isoprostanes and 2,3-dinor-5, 6-dihydro-15- $F_{2 t}$-isoprostane}

At enrolment, participants provided samples of first morning urine during a home visit by the study personnel. Reliability studies have demonstrated that a single morning sample adequately reflects daily excretion of $\mathrm{F}_{2}$-IsoP, with concentrations similar to those obtained from a 24-h urine sample ${ }^{(31)}$. Urinary $\mathrm{F}_{2}$-IsoP and $15-\mathrm{F}_{2 \mathrm{t}}$-IsoP-M were measured using GC/ negative ion chemical ionisation MS at the Eicosanoid Core Laboratory at Vanderbilt University Medical Center. The methods used have been published in detail previously ${ }^{(32-34)}$. The CV for quality control duplicates were $16.0 \%$ for $\mathrm{F}_{2}$-IsoP and $12.5 \%$ for $15-\mathrm{F}_{2 \mathrm{t}}$-IsoP-M. Reported $\mathrm{F}_{2}$-IsoP and $15-\mathrm{F}_{2 \mathrm{t}}$-IsoP-M values were adjusted for creatinine concentrations ( $\mathrm{ng} / \mathrm{mg}$ of creatinine) to correct for urine diluteness ${ }^{(35)}$.

\section{Questionnaire measures}

All dietary and nutrient intakes were ascertained using the Block $98 \mathrm{FFQ}^{(36)}$, completed at enrolment, and refer to average daily intakes in the previous 12 months. Total dietary carotenoids were calculated as the sum of $\beta$-carotene, $\alpha$-carotene, lycopene, lutein + zeaxanthin and cryptoxanthin. Supplement use information was also ascertained from the FFQ and was available for vitamin $\mathrm{E}$, vitamin $\mathrm{C}$, $\beta$-carotene, vitamin $\mathrm{A}$, Se and Zn. Scores on the Healthy Eating Index: 1999-2000, a measure of diet quality developed by the US Department of Agriculture $^{(37)}$, were also calculated from the FFQ.

Physical activity during the previous 12 months was selfreported via a questionnaire completed at enrolment. Participants were asked to report the number of hours per week they spent engaging in specific activities, and weekly energy expenditures were calculated using the metabolic equivalent (MET) values for each activity as listed in established guidelines ${ }^{(38)}$. Total physical activity was estimated by summing the MET-h/week of sports or exercise sessions and daily activities. Alcohol consumption was also self-reported on enrolment through questionnaires. Participants reported their average number of drinks per week. This value was multiplied by 14 (the number of grams of alcohol in a standard drink) and used to calculate an average daily intake of alcohol in grams. Current height and weight, used to calculate BMI $\left(\mathrm{kg} / \mathrm{m}^{2}\right)$, were measured during home visits by trained study personnel at enrolment. Information regarding socio-demographic factors and smoking status was collected at enrolment using questionnaires. We excluded women who were missing an FFQ ( $n$ 18) or who had implausible values for energy intake $(<2092$ or $>20920 \mathrm{~kJ} / \mathrm{d}(<500$ or $>5000 \mathrm{kcal} / \mathrm{d} ; n$ 6)).

\section{Statistical analyses}

Frequencies and percentages were used to describe categorical variables. Medians and quartiles were calculated for continuous variables including dietary intakes, physical activity and alcohol consumption. 
Values of $\mathrm{F}_{2}$-IsoP and $15-\mathrm{F}_{2 \mathrm{t}}$-IsoP-M were highly skewed, and thus were log-transformed to approximate a normal distribution. Using generalised linear models, geometric means of $\mathrm{F}_{2}$-IsoP and $15-\mathrm{F}_{2 \mathrm{t}}$-IsoP-M were calculated by quartiles of all lifestyle variables. Models were adjusted for variables considered a priori as potential confounders. For all exposure variables, geometric means were adjusted for age (continuous), BMI (continuous), race/ethnicity (non-Hispanic white, non-Hispanic black, Hispanic, other), physical activity (total MET-h/week, continuous), household income $(<\$ 20000$, \$20 000-\$49999, \$50000-\$99999, \$100 000-\$200000 and $>\$ 200000$ ) and current smoking status (yes/no). Means according to antioxidant nutrients were further adjusted for total energy intake $(\mathrm{kJ} / \mathrm{d}(\mathrm{kcal} / \mathrm{d})$ continuous), and values according to physical activity, alcohol intake, fruit and vegetable consumption, dietary fats intake and Healthy Eating Index scores were further adjusted for use of any multivitamins and/or supplements (yes/no). For physical activity, alcohol intake and dietary fats, geometric means were additionally adjusted for fruit and vegetable servings per day (continuous). Linear regression models, with continuous, log-transformed $\mathrm{F}_{2}$-IsoP or $15-\mathrm{F}_{2 \mathrm{t}}$-IsoP-M as the dependent variable, were used to evaluate trends. For antioxidant nutrients, we hypothesised that relationships would be approximately linear within the range of values consumed by women in this population. However, to evaluate potential curvilinear trends, we visually assessed scatterplots of all exposures plotted individually against $\mathrm{F}_{2}$-IsoP and $15-\mathrm{F}_{2 \mathrm{t}}$-IsoP-M. In our assessment, no exposure-outcome relationships appeared to be U-shaped. Thus, we proceeded with the evaluation of linear trends using linear regression models. To avoid problems of collinearity in adjusted regression models, related dietary variables were evaluated as covariates individually, rather than in combination.

For analyses of vitamin $\mathrm{E}$, vitamin $\mathrm{C}, \beta$-carotene, vitamin $\mathrm{A}$, Se and $\mathrm{Zn}$ (nutrients for which we had available information on supplement use), we evaluated associations for dietary intakes alone, as well as for combined intakes from both dietary and supplemental sources. In the dietary intake analyses of these nutrients, we performed sensitivity analyses excluding women who reported taking a supplement for that particular nutrient.

In further sensitivity analyses, we excluded women who were current smokers at enrolment. We also performed stratified analyses by BMI (18.5-29.9 v. 30.0 $\left.+\mathrm{kg} / \mathrm{m}^{2}\right)$ to investigate potential effect modification. Tests for statistical interaction were conducted by including cross-product interaction terms in regression models.

The number of missing values was $<5 \%$ for all variables, and therefore missing values were left as missing in all analyses. Two-sided $P$ values $<0.05$ were considered to be statistically significant. All statistical analyses were conducted with Sister Study Data Release 4.0 using SAS 9.4 (SAS Institute).

\section{Results}

The geometric mean concentrations of $\mathrm{F}_{2}-\mathrm{IsoP}$ and $15-\mathrm{F}_{2 \mathrm{t}}-\mathrm{IsoP}-\mathrm{M}$ were $1.44(\mathrm{SD} 0.75)$ and $0.71(\mathrm{sD} 0.32) \mathrm{ng} / \mathrm{mgCr}$, respectively. Log-transformed $\mathrm{F}_{2}$-IsoP and $15-\mathrm{F}_{2 \mathrm{t}}$-IsoP-M values were highly correlated $(r 0.58, P<0 \cdot 001)$. Both $\mathrm{F}_{2}$-IsoP and $15-\mathrm{F}_{2 \mathrm{t}}-\mathrm{IsoP}-\mathrm{M}$ were positively associated with BMI ( $\mathrm{F}_{2}$-IsoP: $r$ 0.25, $P<0 \cdot 001$; 15- $\mathrm{F}_{2 \mathrm{t}}$-IsoP-M: $\left.\quad r \quad 0.37, \quad P<0.001\right)$. Participants were predominately non-Hispanic white $(88 \%)$ with a median age of 47 years and a median BMI of $25.6 \mathrm{~kg} / \mathrm{m}^{2}$ (Table 1 ).

After multivariable adjustment, total MET-h/week of physical activity was inversely associated with $\mathrm{F}_{2}$-IsoP $\left(P_{\text {trend }}=0.003\right)$ (Table 2). A weaker, non-significant trend was observed for $15-\mathrm{F}_{2 \mathrm{t}}$-IsoP-M. Although alcohol intake appeared to be inversely associated with concentrations of $\mathrm{F}_{2}$-IsoP, this association was not statistically significant. Healthy Eating Index scores were not significantly associated with either $\mathrm{F}_{2}$-IsoP or $15-\mathrm{F}_{2 \mathrm{t}}$-IsoP-M in adjusted models. No significant relationships were observed between $\mathrm{F}_{2}$-IsoP or $15-\mathrm{F}_{2 \mathrm{t}}$-IsoP-M and total fat, total dietary $n-6$ fatty acids, total dietary $n-3$ fatty acids, SFA, MUFA, PUFA or total dietary short-chain $n-3$ fatty acids. Intake of total dietary long-chain $n-3$ fatty acids was inversely associated with $15-\mathrm{F}_{2 \mathrm{t}^{-}}$ IsoP-M ( $\left.P_{\text {trend }}=0.03\right)$, but was marginally associated with $\mathrm{F}_{2}$-IsoP $\left(P_{\text {trend }}=0 \cdot 06\right)$. Higher intake of trans fat was associated with higher $\mathrm{F}_{2}$-IsoP $\quad\left(P_{\text {trend }}<0 \cdot 001\right)$ and $15-\mathrm{F}_{2 \mathrm{t}}-\mathrm{IsoP}-\mathrm{M}$ $\left(P_{\text {trend }}=0 \cdot 002\right)$.

Although fruit consumption was inversely associated with $\mathrm{F}_{2^{-}}$ IsoP only $\left(P_{\text {trend }}=0.04\right)$, vegetable consumption was inversely associated with both $\mathrm{F}_{2}$-IsoP $\left(P_{\text {trend }}<0 \cdot 001\right)$ and $15-\mathrm{F}_{2 \mathrm{t}}$-IsoP-M $\left(P_{\text {trend }}<0.001\right)$ (Table 3 ). Inverse associations were observed between vitamin $\mathrm{E}$ and both $\mathrm{F}_{2}$-IsoP $\left(P_{\text {trend }}<0.001\right)$ and $15-\mathrm{F}_{2 \mathrm{t}^{-}}$ IsoP-M $\left(P_{\text {trend }}<0 \cdot 001\right)$. Higher vitamin $\mathrm{C}$ intake was associated with lower $\mathrm{F}_{2}$-IsoP $\left(P_{\text {trend }}=0 \cdot 01\right)$, with a similar, although

Table 1. Participant characteristics(Numbers and percentages; medians and interquartile ranges (IQR); $n$ 888)

\begin{tabular}{|c|c|c|c|}
\hline & $n$ & & $\%$ \\
\hline \multicolumn{4}{|l|}{ Age (years) } \\
\hline Median & \multirow{2}{*}{\multicolumn{3}{|c|}{$\begin{array}{c}47 \\
44,50\end{array}$}} \\
\hline IQR & & & \\
\hline \multicolumn{4}{|l|}{$\mathrm{BMI}\left(\mathrm{kg} / \mathrm{m}^{2}\right)$} \\
\hline Median & \multirow{2}{*}{\multicolumn{3}{|c|}{$\begin{array}{c}25 \cdot 6 \\
22 \cdot 5,30 \cdot 4\end{array}$}} \\
\hline IQR & & & \\
\hline Current smoker & 75 & & 8 \\
\hline \multicolumn{4}{|l|}{ Race/ethnicity } \\
\hline Non-Hispanic white & 778 & & 88 \\
\hline Non-Hispanic black & 55 & & 6 \\
\hline Hispanic & 34 & & 4 \\
\hline Other & 21 & & 2 \\
\hline \multicolumn{4}{|l|}{ Household income } \\
\hline$<\$ 20000$ & 14 & & 2 \\
\hline$\$ 20000-\$ 49000$ & 129 & & 15 \\
\hline$\$ 50000-\$ 99999$ & 364 & & 42 \\
\hline$\$ 100000-\$ 200000$ & 273 & & 31 \\
\hline$>\$ 200000$ & 87 & & 10 \\
\hline \multicolumn{4}{|c|}{ Total energy intake $(\mathrm{kJ} / \mathrm{d})$} \\
\hline Median & \multirow{2}{*}{\multicolumn{3}{|c|}{$\begin{array}{c}6394 \cdot 0 \\
5177 \cdot 7,8230 \cdot 3\end{array}$}} \\
\hline IQR & & & \\
\hline \multicolumn{4}{|c|}{ Total energy intake (kcal/d) } \\
\hline Median & \multirow{2}{*}{\multicolumn{3}{|c|}{$\begin{array}{c}1528 \cdot 2 \\
1237 \cdot 5,1967 \cdot 1\end{array}$}} \\
\hline IQR & & & \\
\hline \multicolumn{4}{|l|}{ Supplement use } \\
\hline Vitamin C & 189 & & 21 \\
\hline Vitamin E & 163 & & 18 \\
\hline$\beta$-Carotene & 22 & & 2 \\
\hline Vitamin A & 25 & & 3 \\
\hline Se & 29 & & 3 \\
\hline $\mathrm{Zn}$ & 68 & & 8 \\
\hline
\end{tabular}


Table 2. Quartiles of physical activity, alcohol and dietary fat subgroups

(Geometric means and $95 \%$ confidence intervals of $\mathrm{F}_{2}$-isoprostanes $\left(\mathrm{F}_{2}\right.$-IsoP) and 2,3-dinor-5,6-dihydro-15- $\mathrm{F}_{2 \mathrm{t}}$-isoprostane $\left(15-\mathrm{F}_{2 \mathrm{t}} \mathrm{I}^{-\mathrm{Iso}} \mathrm{P}-\mathrm{M}\right)$; $n$ 888)

\begin{tabular}{|c|c|c|c|c|c|c|c|c|c|c|c|c|}
\hline & \multicolumn{6}{|c|}{$\mathrm{F}_{2}$-IsoP } & \multicolumn{6}{|c|}{$15-F_{2 t}-$ IsoP-M } \\
\hline & \multicolumn{2}{|c|}{ Unadjusted } & \multirow[b]{2}{*}{$P_{\text {trend }}$} & \multicolumn{2}{|c|}{ Adjusted $†$} & \multirow[b]{2}{*}{$P_{\text {trend }}$} & \multicolumn{2}{|c|}{ Unadjusted } & \multirow[b]{2}{*}{$P_{\text {trend }}$} & \multicolumn{2}{|c|}{ Adjusted $†$} & \multirow[b]{2}{*}{$P_{\text {trend }}$} \\
\hline & Mean & $95 \% \mathrm{Cl}$ & & Mean & $95 \% \mathrm{Cl}$ & & Mean & $95 \% \mathrm{Cl}$ & & Mean & $95 \% \mathrm{Cl}$ & \\
\hline Physical activity (total MET-h/week) & & & $<0.001$ & & & 0.003 & & & $<0.001$ & & & 0.174 \\
\hline$<28 \cdot 12$ & 1.68 & $1.57,1.80$ & & 1.60 & $1.49,1.71$ & & 0.82 & $0.77,0.87$ & & 0.77 & $0.72,0.81$ & \\
\hline $28 \cdot 12-44 \cdot 19$ & 1.40 & $1.31,1.50$ & & 1.41 & $1.32,1.51$ & & 0.70 & $0.66,0.74$ & & 0.70 & $0.66,0.73$ & \\
\hline $44 \cdot 20-65 \cdot 88$ & 1.44 & $1.35,1.54$ & & 1.46 & $1.37,1.56$ & & 0.70 & $0.66,0.74$ & & 0.70 & $0.66,0.74$ & \\
\hline$\geq 65.89$ & $1 \cdot 26$ & $1.17,1.35$ & & $1 \cdot 33$ & $1 \cdot 24,1.42$ & & 0.64 & $0.61,0.68$ & & 0.69 & $0.65,0.72$ & \\
\hline Alcohol intake $(\mathrm{g} / \mathrm{d})$ & & & 0.014 & & & 0.086 & & & 0.579 & & & 0.294 \\
\hline$<0.23$ & 1.58 & $1.47,1.69$ & & 1.56 & $1.46,1.67$ & & 0.79 & $0.74,0.84$ & & 0.75 & $0.71,1.80$ & \\
\hline $0.23-1.84$ & 1.50 & 1.391 .61 & & 1.45 & $1.35,1.55$ & & 0.73 & $0.68,0.77$ & & 0.70 & $0.66,0.74$ & \\
\hline $1.85-7.99$ & 1.38 & $1.29,1.48$ & & 1.39 & $1.30,1.49$ & & 0.66 & $0.63,0.70$ & & 0.68 & $0.64,0.71$ & \\
\hline$\geq 8.0$ & $1 \cdot 33$ & $1.25,1.42$ & & 1.40 & $1.31,1.49$ & & 0.68 & $0.65,0.72$ & & 0.72 & $0.69,0.76$ & \\
\hline Total dietary $n-6$ fatty acids (g) & & & 0.451 & & & 0.654 & & & 0.726 & & & 0.445 \\
\hline$<9.43$ & 1.43 & $1.33,1.53$ & & 1.43 & $1.34,1.53$ & & 0.73 & $0.69,0.78$ & & 0.72 & $0.69,0.77$ & \\
\hline $9 \cdot 43-12.63$ & 1.47 & $1.37,1.57$ & & 1.50 & $1.40,1.60$ & & 0.68 & $0.64,0.72$ & & $0 \cdot 70$ & $0.66,0.74$ & \\
\hline $12 \cdot 64-17 \cdot 43$ & 1.48 & $1.39,1.59$ & & 1.47 & $1.37,1.57$ & & 0.72 & $0.68,0.77$ & & 0.72 & $0.68,0.76$ & \\
\hline$\geq 17 \cdot 44$ & 1.37 & $1.28,1.47$ & & 1.39 & $1 \cdot 30,1.49$ & & 0.71 & $0.67,0.75$ & & $0 \cdot 70$ & $0.66,0.74$ & \\
\hline Total dietary $n-3$ fatty acids (g) & & & 0.306 & & & 0.604 & & & 0.409 & & & 0.226 \\
\hline$<1.05$ & 1.45 & $1.35,1.55$ & & 1.43 & $1.34,1.54$ & & 0.75 & $0.71,0.79$ & & 0.73 & $0.69,0.78$ & \\
\hline $1.05-1.43$ & 1.52 & $1.41,1.62$ & & 1.52 & $1.43,1.63$ & & 0.70 & $0.66,0.75$ & & 0.71 & $0.68,0.75$ & \\
\hline $1.44-1.93$ & 1.41 & $1.32,1.51$ & & 1.42 & $1.33,1.51$ & & 0.69 & $0.65,0.74$ & & 0.70 & $0.66,0.74$ & \\
\hline$\geq 1.94$ & $1 \cdot 38$ & $1.29,1.48$ & & 1.41 & $1 \cdot 32,1.51$ & & 0.70 & $0.66,0.75$ & & $0 \cdot 70$ & $0.66,0.74$ & \\
\hline SFA $(g)$ & & & 0.091 & & & 0.358 & & & 0.052 & & & 0.564 \\
\hline$<13.63$ & 1.39 & $1.30,1.49$ & & 1.46 & $1.36,1.56$ & & 0.70 & $0.66,0.74$ & & 0.72 & $0.68,0.76$ & \\
\hline $13 \cdot 63-18 \cdot 50$ & 1.42 & $1.33,1.52$ & & 1.43 & $1.34,1.53$ & & 0.68 & $0.64,0.72$ & & 0.69 & $0.65,0.72$ & \\
\hline $18 \cdot 51-25.05$ & 1.40 & $1.31,1.50$ & & 1.39 & $1.30,1.49$ & & 0.73 & $0.68,0.77$ & & 0.72 & $0.68,0.76$ & \\
\hline$\geq 25.06$ & 1.53 & $1.43,1.64$ & & 1.51 & $1.41,1.61$ & & 0.75 & $0.71,0.79$ & & 0.72 & $0.68,0.76$ & \\
\hline MUFA (g) & & & 0.678 & & & 0.964 & & & 0.858 & & & 0.667 \\
\hline$<18.06$ & 1.44 & $1.35,1.55$ & & 1.46 & $1 \cdot 36,1.56$ & & 0.72 & $0.68,0.77$ & & 0.72 & $0.68,0.76$ & \\
\hline $18 \cdot 06-24 \cdot 37$ & $1 \cdot 38$ & $1 \cdot 29,1.48$ & & 1.40 & $1.31,1.50$ & & 0.68 & $0.64,0.72$ & & 0.70 & $0.66,0.73$ & \\
\hline $24 \cdot 38-32 \cdot 86$ & 1.47 & $1.37,1.57$ & & 1.46 & $1.37,1.56$ & & 0.72 & $0.68,0.77$ & & 0.72 & $0.69,0.76$ & \\
\hline$\geq 32.87$ & 1.45 & $1.36,1.56$ & & 1.47 & $1.37,1.57$ & & 0.72 & $0.68,0.76$ & & $0 \cdot 70$ & $0.67,0.75$ & \\
\hline PUFA (g) & & & 0.469 & & & 0.676 & & & 0.736 & & & 0.434 \\
\hline$<10.67$ & 1.42 & $1.33,1.52$ & & 1.43 & $1.34,1.53$ & & 0.73 & $0.69,0.77$ & & 0.72 & $0.68,0.77$ & \\
\hline $10 \cdot 67-14 \cdot 27$ & 1.48 & $1.38,1.59$ & & 1.51 & $1.41,1.61$ & & 0.68 & $0.64,0.72$ & & 0.69 & $0.66,0.73$ & \\
\hline $14 \cdot 28-19 \cdot 49$ & 1.48 & $1.39,1.58$ & & 1.46 & $1.37,1.56$ & & 0.72 & $0.68,0.77$ & & 0.72 & $0.68,0.76$ & \\
\hline$\geq 19.50$ & $1 \cdot 37$ & $1.28,1.46$ & & $1 \cdot 38$ & $1 \cdot 29,1.48$ & & 0.71 & $0.67,0.76$ & & 0.71 & $0.67,0.75$ & \\
\hline Trans fat $(\mathrm{g})$ & & & $<0.001$ & & & $<0.001$ & & & $<0.001$ & & & 0.002 \\
\hline$<2.94$ & $1 \cdot 26$ & $1 \cdot 18,1.35$ & & 1.33 & $1 \cdot 25,1.42$ & & 0.64 & $0.61,0.68$ & & 0.67 & $0.63,0.70$ & \\
\hline $2 \cdot 94-4 \cdot 36$ & $1 \cdot 37$ & $1.28,1.47$ & & 1.40 & $1.31,1.49$ & & 0.67 & $0.64,0.72$ & & 0.69 & $0.65,0.73$ & \\
\hline $4 \cdot 37-6 \cdot 24$ & 1.50 & $1.41,1.61$ & & 1.48 & $1.39,1.58$ & & 0.74 & $0.70,0.78$ & & 0.73 & $0.69,0.77$ & \\
\hline$\geq 6 \cdot 25$ & 1.64 & $1.53,1.75$ & & 1.58 & $1.48,1.69$ & & 0.80 & $0.75,0.85$ & & 0.76 & $0.72,0.80$ & \\
\hline Total fat (g) & & & 0.863 & & & 0.860 & & & 0.637 & & & 0.832 \\
\hline$<46.54$ & 1.43 & $1.34,1.53$ & & 1.45 & $1 \cdot 36,1.56$ & & 0.71 & $0.67,0.76$ & & 0.71 & $0.68,0.76$ & \\
\hline $46 \cdot 55-62 \cdot 39$ & 1.40 & $1.31,1.50$ & & 1.42 & $1.33,1.51$ & & 0.70 & $0.66,0.74$ & & 0.71 & $0.67,0.75$ & \\
\hline $62 \cdot 40-84 \cdot 37$ & 1.46 & $1.36,1.56$ & & 1.47 & $1.37,1.56$ & & 0.71 & $0.67,0.76$ & & 0.72 & $0.68,0.76$ & \\
\hline$\geq 84.38$ & 1.45 & $1.36,1.56$ & & 1.45 & $1.35,1.55$ & & 0.72 & $0.68,0.77$ & & $0 \cdot 70$ & $0.66,0.74$ & \\
\hline Total dietary short-chain $n-3$ fatty acids (g) & & & 0.629 & & & 0.824 & & & 0.782 & & & 0.369 \\
\hline$<0.96$ & 1.45 & $1.35,1.55$ & & 1.44 & $1 \cdot 34,1.54$ & & 0.73 & $0.68,0.77$ & & 0.71 & $0.67,0.75$ & \\
\hline $0.96-1.32$ & 1.52 & $1.42,1.62$ & & 1.53 & $1.43,1.64$ & & 0.71 & $0.67,0.76$ & & 0.72 & $0.68,0.76$ & \\
\hline $1 \cdot 33-1 \cdot 78$ & $1 \cdot 38$ & $1.29,1.48$ & & $1 \cdot 39$ & $1 \cdot 30,1.48$ & & 0.69 & $0.65,0.73$ & & 0.69 & $0.66,0.73$ & \\
\hline$\geq 1.79$ & 1.41 & $1.32,1.51$ & & 1.43 & $1 \cdot 34,1.54$ & & 0.72 & $0.68,0.76$ & & $0 \cdot 71$ & $0.67,0.76$ & \\
\hline Total dietary long-chain $n-3$ fatty acids (g) & & & $<0.001$ & & & 0.063 & & & $<0.001$ & & & 0.027 \\
\hline$<0.05$ & 1.53 & $1.43,1.64$ & & 1.49 & $1.40,1.60$ & & 0.77 & $0.73,0.82$ & & 0.75 & $0.71,0.79$ & \\
\hline $0.05-0.08$ & 1.54 & $1.44,1.65$ & & 1.50 & $1.41,1.60$ & & 0.74 & $0.70,0.79$ & & 0.73 & $0.69,0.77$ & \\
\hline $0.09-0.15$ & $1 \cdot 37$ & $1.28,1.47$ & & 1.41 & $1.31,1.50$ & & 0.69 & $0.65,0.73$ & & $0 \cdot 70$ & $0.66,0.74$ & \\
\hline$\geq 0 \cdot 16$ & $1 \cdot 32$ & $1 \cdot 23,1 \cdot 41$ & & $1 \cdot 39$ & $1 \cdot 29,1 \cdot 48$ & & 0.65 & $0.62,0.69$ & & 0.68 & $0.64,0.71$ & \\
\hline Healthy Eating Index Scoreł & & & $<0.001$ & & & 0.361 & & & $<0.001$ & & & 0.429 \\
\hline$<53$ & 1.53 & $1.43,1.65$ & & 1.47 & $1.37,1.57$ & & 0.77 & $0.73,0.82$ & & 0.72 & $0.68,0.76$ & \\
\hline $53-62$ & 1.54 & $1.44,1.65$ & & 1.51 & $1.41,1.61$ & & 0.75 & $0.71,0.79$ & & 0.73 & $0.69,0.77$ & \\
\hline $63-71$ & 1.35 & $1.26,1.44$ & & 1.39 & $1 \cdot 30,1.49$ & & 0.68 & $0.64,0.72$ & & 0.70 & $0.66,0.74$ & \\
\hline$\geq 72$ & $1 \cdot 34$ & $1.26,1.44$ & & 1.42 & $1 \cdot 32,1.52$ & & 0.66 & $0.62,0.70$ & & 0.70 & $0.66,0.74$ & \\
\hline
\end{tabular}

MET, metabolic equivalent.

* Values of $\mathrm{F}_{2}$-IsoP and $15-\mathrm{F}_{2 \mathrm{t}}$-IsoP-M are expressed in $\mathrm{ng} / \mathrm{mg}$ creatinine.

$\dagger$ Adjusted for age, BMI, race/ethnicity, physical activity, household income, current smoking status, fruit and vegetable servings per day, and any multivitamin and/or supplement use.

‡ Adjusted for age, BMI, race/ethnicity, physical activity, household income, current smoking status, and any multivitamin and/or supplement use 
Table 3. Quartiles of fruit and vegetable servings and intake of dietary antioxidant nutrients*

(Geometric means and $95 \%$ confidence intervals of $\mathrm{F}_{2}$-isoprostanes $\left(\mathrm{F}_{2}\right.$-IsoP) and 2,3-dinor-5,6-dihydro-15- $\mathrm{F}_{2 \mathrm{t}}$-isoprostane $\left(15-\mathrm{F}_{2 \mathrm{t}}{ }^{-}\right.$-IsoP-M); $n$ 888)

\begin{tabular}{|c|c|c|c|c|c|c|c|c|c|c|c|c|}
\hline & \multicolumn{6}{|c|}{$\mathrm{F}_{2}$-IsoP } & \multicolumn{6}{|c|}{$15-F_{2 t}$-IsoP-M } \\
\hline & \multicolumn{2}{|c|}{ Unadjusted } & \multirow[b]{2}{*}{$P_{\text {trend }}$} & \multicolumn{2}{|c|}{ Adjusted $\dagger$} & \multirow[b]{2}{*}{$P_{\text {trend }}$} & \multicolumn{2}{|c|}{ Unadjusted } & \multirow[b]{2}{*}{$P_{\text {trend }}$} & \multicolumn{2}{|c|}{ Adjusted $\dagger$} & \\
\hline & Mean & $95 \% \mathrm{Cl}$ & & Mean & $95 \% \mathrm{Cl}$ & & Mean & $95 \% \mathrm{Cl}$ & & Mean & $95 \% \mathrm{Cl}$ & $P_{\text {trend }}$ \\
\hline Fruits (servings/d) & & & $<0.001$ & & & 0.035 & & & $<0.001$ & & & 0.365 \\
\hline$<0.6$ & 1.60 & $1.49,1.72$ & & 1.53 & $1.42,1.64$ & & 0.77 & $0.73,0.82$ & & 0.73 & $0.69,0.77$ & \\
\hline $0.6-1.0$ & 1.51 & $1.41,1.62$ & & 1.51 & $1.41,1.61$ & & 0.73 & $0.69,0.78$ & & 0.72 & $0.68,0.76$ & \\
\hline $1.1-1.9$ & 1.40 & $1.30,1.50$ & & 1.41 & $1.32,1.51$ & & 0.72 & $0.67,0.76$ & & 0.73 & $0.69,0.77$ & \\
\hline$\geq 2.0$ & 1.29 & $1.21,1.37$ & & 1.36 & $1.28,1.45$ & & 0.65 & $0.62,0.69$ & & 0.68 & $0.65,0.72$ & \\
\hline Vegetables (servings/d) & & & $<0.001$ & & & $<0.001$ & & & $<0.001$ & & & $<0.001$ \\
\hline$<1.6$ & 1.64 & $1.53,1.76$ & & 1.64 & $1.53,1.77$ & & $0 \cdot 76$ & $0.71,0.81$ & & 0.75 & $0.71,0.80$ & \\
\hline $1.6-2.6$ & 1.44 & $1.35,1.54$ & & 1.47 & $1.37,1.56$ & & 0.76 & $0.72,0.80$ & & 0.76 & $0.72,0.80$ & \\
\hline $2 \cdot 7-4 \cdot 2$ & 1.40 & $1.31,1.50$ & & 1.40 & $1.31,1.50$ & & 0.68 & $0.64,0.72$ & & 0.67 & $0.64,0.71$ & \\
\hline$\geq 4.3$ & 1.31 & $1.22,1.40$ & & 1.31 & $1.23,1.41$ & & 0.66 & $0.63,0.70$ & & 0.67 & $0.63,0.71$ & \\
\hline Vitamin E (a-tocopherol, mg) & & & $<0.001$ & & & $<0.001$ & & & 0.006 & & & $<0.001$ \\
\hline$<5.6$ & 1.55 & $1.45,1.66$ & & 1.66 & $1.53,1.80$ & & 0.78 & $0.74,0.83$ & & 0.83 & $0.77,0.88$ & \\
\hline $5 \cdot 6-7.5$ & 1.43 & $1.34,1.53$ & & 1.49 & $1.39,1.59$ & & 0.70 & $0.66,0.74$ & & 0.72 & $0.68,0.76$ & \\
\hline $7 \cdot 6-10 \cdot 0$ & 1.46 & $1.37,1.56$ & & 1.44 & $1.35,1.54$ & & 0.71 & $0.67,0.75$ & & 0.70 & $0.67,0.74$ & \\
\hline$\geq 10.1$ & 1.30 & $1.23,1.41$ & & 1.24 & $1.14,1.34$ & & 0.67 & $0.63,0.71$ & & 0.62 & $0.58,0.66$ & \\
\hline Vitamin C (mg) & & & $<0.001$ & & & 0.010 & & & 0.008 & & & 0.130 \\
\hline$<55.4$ & 1.60 & $1.49,1.71$ & & 1.58 & $1.47,1.69$ & & 0.77 & $0.73,0.82$ & & 0.76 & $0.71,0.80$ & \\
\hline $55 \cdot 4-84.1$ & 1.47 & $1.37,1.57$ & & 1.49 & $1.39,1.59$ & & 0.74 & $0.70,0.79$ & & 0.73 & $0.70,0.78$ & \\
\hline $84 \cdot 2-121 \cdot 7$ & 1.39 & $1.30,1.49$ & & 1.43 & $1.33,1.52$ & & 0.67 & $0.63,0.71$ & & 0.68 & $0.65,0.72$ & \\
\hline$\geq 121.8$ & 1.30 & $1.22,1.39$ & & 1.30 & $1.21,1.40$ & & 0.67 & $0.64,0.71$ & & 0.67 & $0.63,0.71$ & \\
\hline$\beta$-Carotene $(\mu \mathrm{g})$ & 1.00 & & $<0.001$ & 1.00 & & $<0.001$ & & & $<0.001$ & & & $<0.001$ \\
\hline$<2448.50$ & 1.62 & $1.52,1.74$ & & 1.59 & $1.49,1.71$ & & 0.80 & $0.75,0.85$ & & 0.78 & $0.74,0.83$ & \\
\hline $2448 \cdot 50-41$ & 1.48 & $1.38,1.58$ & & 1.50 & $1.40,1.60$ & & 0.75 & $0.71,0.79$ & & 0.75 & $0.71,0.79$ & \\
\hline $4111.90-69$ & 1.42 & $1.33,1.52$ & & 1.44 & $1.35,1.54$ & & 0.67 & $0.64,0.71$ & & 0.67 & $0.64,0.71$ & \\
\hline$\geq 6999.75$ & $1 \cdot 26$ & $1.17,1.34$ & & 1.27 & $1.19,1.36$ & & 0.64 & $0.60,0.68$ & & 0.65 & $0.61,0.68$ & \\
\hline Vitamin A (RAE) & & & $<0.001$ & & & $<0.001$ & & & 0.001 & & & $<0.001$ \\
\hline$<543.40$ & 1.58 & $1.47,1.69$ & & 1.60 & $1.49,1.72$ & & 0.78 & $0.73,0.83$ & & 0.78 & $0.74,0.83$ & \\
\hline $543 \cdot 40-745 \cdot 79$ & 1.44 & $1.34,1.54$ & & 1.47 & $1.38,1.58$ & & 0.72 & $0.67,0.76$ & & 0.72 & $0.68,0.76$ & \\
\hline $745 \cdot 80$ & 1.47 & $1.38,1.58$ & & 1.48 & $1.38,1.58$ & & 0.70 & $0.66,0.74$ & & 0.70 & $0.66,0.74$ & \\
\hline$\geq 1074.6$ & 1.28 & $1 \cdot 19,1.37$ & & 1.26 & $1.17,1.35$ & & 0.66 & $0.62,0.70$ & & 0.65 & $0.61,0.69$ & \\
\hline $\operatorname{Se}(\mu \mathrm{g})$ & & & 0.434 & & & $<0.001$ & & & 0.961 & & & $<0.001$ \\
\hline$<60 \cdot 10$ & 1.43 & $1.34,1.53$ & & 1.54 & $1.41,1.67$ & & 0.73 & $0.69,0.77$ & & 0.77 & $0.72,0.83$ & \\
\hline $60.10-78.59$ & 1.46 & $1.36,1.56$ & & 1.49 & $1.39,1.60$ & & 0.69 & $0.65,0.73$ & & 0.71 & $0.67,0.75$ & \\
\hline 78.60 & 1.46 & $1.36,1.56$ & & 1.45 & $1.36,1.55$ & & 0.72 & $0.68,0.76$ & & 0.71 & $0.67,0.75$ & \\
\hline$\geq 103.7$ & 1.40 & $1.31,1.50$ & & 1.32 & $1.20,1.44$ & & 0.71 & $0.67,0.76$ & & 0.66 & $0.61,0.71$ & \\
\hline $\mathrm{Zn}(\mathrm{mg})$ & & & 0.085 & & & 0.010 & & & 0.571 & & & 0.073 \\
\hline$<7.0$ & 1.48 & 1.59 & & 1.58 & $1.45,1.71$ & & 0.74 & 0.79 & & 0.79 & $0.74,0.84$ & \\
\hline $7 \cdot 0-9 \cdot 4$ & 1.48 & $1.38,1.58$ & & 1.53 & $1.43,1.64$ & & 0.72 & $0.68,0.76$ & & 0.74 & $0.70,0.78$ & \\
\hline $9 \cdot 5-12 \cdot 5$ & 1.40 & $1.31,1.50$ & & 1.38 & $1.30,1.48$ & & 0.69 & $0.66,0.74$ & & 0.68 & $0.65,0.72$ & \\
\hline$\geq 12.6$ & 1.39 & $1.30,1.48$ & & 1.32 & $1.21,1.43$ & & 0.70 & $0.66,0.74$ & & 0.64 & $0.60,0.69$ & \\
\hline Lutein + zeaxanthir & & & $<0.001$ & & & $<0.001$ & & & $<0.001$ & & & $<0.001$ \\
\hline$<1927.95$ & 1.67 & $1.57,1.79$ & & 1.64 & $1.53,1.76$ & & 0.82 & $0.77,0.87$ & & 0.80 & $0.76,0.85$ & \\
\hline $1927 \cdot 95-32$ & 1.42 & $1.33,1.52$ & & 1.44 & $1.35,1.54$ & & 0.72 & $0.68,0.77$ & & 0.73 & $0.69,0.77$ & \\
\hline $3299.90-6159.49$ & 1.45 & $1.35,1.55$ & & 1.48 & $1.39,1.58$ & & 0.70 & $0.66,0.74$ & & 0.70 & $0.67,0.74$ & \\
\hline$\geq 6159.50$ & 1.24 & $1.16,1.33$ & & 1.25 & $1.16,1.34$ & & 0.62 & $0.59,0.66$ & & 0.63 & $0.59,0.66$ & \\
\hline Lycopene $(\mu \mathrm{g})$ & & & 0.986 & & & 0.487 & & & 0.145 & & & 0.514 \\
\hline$<2570.50$ & 1.44 & $1.34,1.54$ & & 1.46 & $1.35,1.57$ & & 0.72 & $0.68,0.76$ & & 0.72 & $0.68,0.77$ & \\
\hline 257 & 1.38 & $1.29,1.48$ & & 1.41 & $1.32,1.51$ & & 0.69 & & & 0.70 & & \\
\hline 5099.94 & 1.54 & $1.44,1.65$ & & 1.56 & $1.46,1.66$ & & 0.71 & .75 & & 0.71 & $0.67,0.75$ & \\
\hline$\geq 6099.94$ & 1.39 & $1.30,1.49$ & & 1.36 & $1.27,1.47$ & & 0.73 & $0.69,0.78$ & & 0.71 & $0.67,0.76$ & \\
\hline$a-\overline{C a r o t e n e}(\mu \mathrm{g})$ & & & 0.012 & & & 0.025 & & & 0.842 & & & 0.510 \\
\hline$<238.40$ & 1.49 & $1.39,1.59$ & & 1.48 & $1.38,1.58$ & & 0.74 & $0.70,0.79$ & & 0.73 & $0.69,0.78$ & \\
\hline $238.40-442.19$ & 1.54 & $1.44,1.65$ & & 1.53 & $1.43,1.64$ & & 0.72 & $0.68,0.76$ & & 0.71 & $0.67,0.75$ & \\
\hline $442 \cdot 20-839 \cdot 49$ & $1 \cdot 36$ & $1.27,1.46$ & & 1.39 & $1.30,1.49$ & & 0.70 & $0.66,0.74$ & & 0.71 & $0.67,0.75$ & \\
\hline$\geq 839.50$ & 1.36 & $1.27,1.46$ & & 1.39 & $1.29,1.49$ & & 0.69 & $0.65,0.73$ & & 0.69 & $0.65,0.73$ & \\
\hline Cryptoxanthin $(\mu \mathrm{g})$ & & & 0.019 & & & 0.379 & & & 0.201 & & & 0.636 \\
\hline$<66.30$ & 1.60 & $1.49,1.71$ & & 1.55 & $1.44,1.66$ & & 0.78 & $0.74,0.83$ & & 0.75 & $0.71,0.80$ & \\
\hline $66 \cdot 30-122.59$ & 1.45 & 1.56 & & 1.45 & 1.3 & & 0.72 & $0.68,0.76$ & & 0.71 & $0.67,0.75$ & \\
\hline $122.60-211.39$ & 1.37 & $1.28,1.47$ & & 1.41 & $1.32,1.50$ & & 0.68 & $0.64,0.72$ & & 0.70 & $0.66,0.74$ & \\
\hline$\geq 211.40$ & 1.34 & $1.25,1.44$ & & 1.38 & $1.29,1.48$ & & 0.68 & $0.64,0.72$ & & 0.69 & $0.65,0.73$ & \\
\hline Total carotenoids $(\mu \mathrm{g})$ & & & $<0.001$ & & & $<0.001$ & & & $<0.001$ & & & $<0.001$ \\
\hline$<8567.9$ & 1.58 & 1.70 & & 1.60 & $1.49,1.72$ & & 0.78 & $0.73,0.82$ & & 0.78 & $0.74,0.83$ & \\
\hline $8567 \cdot 9-129$ & 1.48 & $1.38,1.58$ & & 1.50 & $1.41,1.60$ & & 0.74 & $0.70,0.78$ & & 0.74 & $0.70,0.78$ & \\
\hline $12989 \cdot 6-20071 \cdot 8$ & 1.43 & $1.33,1.53$ & & 1.46 & $1.36,1.56$ & & 0.68 & $0.64,0.72$ & & 0.68 & $0.65,0.72$ & \\
\hline$\geq 20071.9$ & 1.27 & $1 \cdot 19,1.36$ & & 1.25 & $1.17,1.34$ & & 0.66 & $0.62,0.70$ & & 0.65 & $0.61,0.69$ & \\
\hline
\end{tabular}

RAE, retinol activity equivalents.

* Values of $\mathrm{F}_{2}$-IsoP and $15-\mathrm{F}_{2 t}$-IsoP-M are expressed in $\mathrm{ng} / \mathrm{mg}$ creatinine.

† Adjusted for age, BMI, race/ethnicity, physical activity, total energy intake, household income and current smoking status; fruit and vegetable servings additionally adjusted for any multivitamin/supplement use. 
non-significant, association with $15-\mathrm{F}_{2 \mathrm{t}}-\mathrm{IsoP}-\mathrm{M} \quad\left(P_{\text {trend }}=0 \cdot 1\right)$. $\beta$-Carotene was inversely associated with $\mathrm{F}_{2}$-IsoP $\left(P_{\text {trend }}<\right.$ $0.001)$ and $15-\mathrm{F}_{2 \mathrm{t}}$-IsoP-M $\left(P_{\text {trend }}<0 \cdot 001\right)$. Similar associations were observed for vitamin A with both $\mathrm{F}_{2}$-IsoP $\left(P_{\text {trend }}<0.001\right)$ and $15-\mathrm{F}_{2 \mathrm{t}}$-IsoP-M $\left(P_{\text {trend }}<0 \cdot 001\right)$. Se intake was inversely associated with $\mathrm{F}_{2}$-IsoP $\left(P_{\text {trend }}<0.001\right)$ and $15-\mathrm{F}_{2 \mathrm{t}}-\mathrm{IsoP}-\mathrm{M}$ $\left(P_{\text {trend }}<0 \cdot 001\right)$. Higher $\mathrm{Zn}$ intake was associated with lower $\mathrm{F}_{2^{-}}$ IsoP $\left(P_{\text {trend }}=0 \cdot 01\right)$ and marginally associated with lower $15-\mathrm{F}_{2 \mathrm{t}^{-}}$ IsoP-M $\left(P_{\text {trend }}=0 \cdot 07\right)$. Strong inverse associations were found between lutein + zeaxanthin and both $\mathrm{F}_{2}$-IsoP $\left(P_{\text {trend }}<0.001\right)$ and $15-\mathrm{F}_{2 \mathrm{t}}$-IsoP-M $\left(P_{\text {trend }}<0 \cdot 001\right)$, although lycopene was not associated with either $\mathrm{F}_{2}$-IsoP or $15-\mathrm{F}_{2 \mathrm{t}}$-IsoP-M. $\alpha$-Carotene was inversely associated with $\mathrm{F}_{2}$-IsoP $\left(P_{\text {trend }}=0.03\right)$ but not significantly associated with $15-\mathrm{F}_{2 \mathrm{t}}-\mathrm{IsoP}-\mathrm{M}\left(P_{\text {trend }}=0 \cdot 5\right)$. Cryptoxanthin was not associated with $\mathrm{F}_{2}$-IsoP or $15-\mathrm{F}_{2 \mathrm{t}}$-IsoP-M. Total carotenoid intake was strongly associated with both $\mathrm{F}_{2}$-IsoP $\left(P_{\text {trend }}<0.001\right)$ and $15-\mathrm{F}_{2 \mathrm{t}}$-IsoP-M $\left(P_{\text {trend }}<0.001\right)$. For dietary vitamin $\mathrm{E}$, vitamin $\mathrm{C}, \beta$-carotene, vitamin $\mathrm{A}$, Se and $\mathrm{Zn}$, patterns remained similar when supplement users for these nutrients were excluded (data not shown).

Associations of $\mathrm{F}_{2}$-IsoP and $15-\mathrm{F}_{2 \mathrm{t}}-\mathrm{IsoP}-\mathrm{M}$ with combined dietary and supplemental intakes of vitamin $\mathrm{E}$, vitamin $\mathrm{C}$, $\beta$-carotene, vitamin $\mathrm{A}$, Se and $\mathrm{Zn}$ were generally similar to those observed for dietary intakes of these nutrients alone (online Supplementary Table S1). However, associations with combined dietary and supplemental sources appeared to be somewhat weaker for vitamin $\mathrm{E}$ and stronger for vitamin $\mathrm{C}$ and $\mathrm{Zn}$, compared with associations with dietary intakes alone.

All trends remained similar when analyses were restricted to non-smokers (data not shown). In stratified analyses, patterns were largely similar between those with a BMI of 18.5 $29.9 \mathrm{~kg} / \mathrm{m}^{2}$ and those with a BMI of $30.0 \mathrm{~kg} / \mathrm{m}^{2}$ or greater. Although inverse associations between $\mathrm{F}_{2}$-IsoP $\left(P_{\text {trend }}=0.002\right)$ and $15-\mathrm{F}_{2 \mathrm{t}}$-IsoP-M $\left(P_{\text {trend }}=0.03\right)$ and physical activity were only apparent among women with a BMI of $18.5-29.9 \mathrm{~kg} / \mathrm{m}^{2}$, the interaction test was not statistically significant $\left(\mathrm{F}_{2}\right.$-IsoP: $P_{\text {interaction }}=0.9 ; 15-\mathrm{F}_{2 \mathrm{t}}$-IsoP-M: $P_{\text {interaction }}=0 \cdot 4 ;$ online Supplementary Table S2). Likewise, $\mathrm{Zn}$ intake was inversely associated with $\mathrm{F}_{2}$-IsoP $\left(P_{\text {trend }}=0.008\right)$ and $15-\mathrm{F}_{2 \mathrm{t}}$-IsoP-M $\left(P_{\text {trend }}=0.03\right)$ only among women with a BMI of $18.5-29.9 \mathrm{~kg} / \mathrm{m}^{2}$, although the interactions were not significant $\left(\mathrm{F}_{2}\right.$-IsoP: $P_{\text {interaction }}=0 \cdot 3$; 15- $\mathrm{F}_{2 \mathrm{t}}$-IsoP-M: $\left.\quad P_{\text {interaction }}=0 \cdot 4\right)$. Fruit intake was inversely associated with $\mathrm{F}_{2} \mathrm{IsoP}$ only among women with a BMI of $18.5-29.9 \mathrm{~kg} / \mathrm{m}^{2}\left(P_{\text {trend }}=0.009\right)$. However, the interaction test did not indicate a significant difference according to BMI $\left(P_{\text {interaction }}=0 \cdot 4 ;\right.$ online Supplementary Table S3).

\section{Discussion}

In this study, we found that oxidative stress, as measured by $\mathrm{F}_{2}$-isoprostane and its metabolite, was associated with a number of dietary and lifestyle factors. Lower oxidative stress was observed with greater intake of fruits and vegetables, antioxidant nutrients and long-chain $n$ - 3 fatty acids, whereas higher oxidative stress was found among women with a greater intake of trans fats. In addition, our findings suggest a possible inverse relationship between total physical activity and oxidative stress. Associations were similar for non-obese and obese women and remained largely unchanged when current smokers were excluded.

Individual nutrients most strongly associated with both $\mathrm{F}_{2}$-IsoP and $15-\mathrm{F}_{2 \mathrm{t}}$-IsoP-M in the present study included vitamin $\mathrm{E}$ and the carotenoids $\beta$-carotene and lutein + zeaxanthin - findings consistent with the antioxidant properties of these compounds. Vitamin E, or $\alpha$-tocopherol, is a lipidsoluble, chain-breaking antioxidant, whereas carotenoids are lipid-soluble compounds that scavenge singlet oxygen ${ }^{(16)}$. Other studies of $\mathrm{F}_{2}$-IsoP have observed similar strong associations with these antioxidant nutrients. A recent cross-sectional study among healthy, middle-aged men found that $\beta$-carotene and lutein + zeaxanthin were the carotenoids with the strongest inverse associations with urinary $\mathrm{F}_{2}$-IsoP ${ }^{(14)}$. The results from the Study of Women's Health Across the Nation (SWAN) showed dietary intakes of vitamin $\mathrm{E}$, lutein and $\beta$-carotene, as well as vitamin A and vitamin $\mathrm{C}$, to be negatively correlated with $\mathrm{F}_{2}$-IsoP ${ }^{(13)}$. The relative strengths of associations with the antioxidant nutrients we evaluated may be partly explained by their efficiency in reacting with various free radicals and pro-oxidants and their ability to interact with other antioxidants $^{(10,39)}$. Tocopherols (vitamin E), for example, are the most abundant and efficient scavengers of peroxyl radicals in biological membranes, and their antioxidant activity is supported by their interaction with vitamin $\mathrm{C}^{(10)}$.

Our findings regarding antioxidant nutrients likely explain, in large part, the inverse associations observed for fruits and vegetables, foods rich in carotenoids and other antioxidants. Although adjustment for multivitamin/supplement use, physical activity, BMI and other confounding factors attenuated associations with fruit intake in our sample, this was not the case for vegetable intake. A stronger trend for vegetables than for fruits in relation to urinary $\mathrm{F}_{2}$-IsoP has been observed previously ${ }^{(13)}$, and may be explained by the specific types of fruits and vegetables commonly consumed among women of this age group, or by the greater range of vegetable servings consumed in this population (0-15), relative to fruit servings (0-5).

We also found that dietary $\mathrm{Zn}$ intake was inversely associated with $\mathrm{F}_{2}$-IsoP, with a similar but non-significant association with 15- $\mathrm{F}_{2 \mathrm{t}}$-IsoP-M. A similar association with $\mathrm{F}_{2}$-IsoP was found among participants in SWAN ${ }^{(13)}$. The antioxidant activity of $\mathrm{Zn}$, a ubiquitous trace element in the body, is proposed to occur through several different mechanisms, one of which involves its role as a cofactor for superoxide dismutase, an important component of antioxidant defence ${ }^{(40)}$. In our sample, associations between $\mathrm{F}_{2}$-IsoP and $15-\mathrm{F}_{2 \mathrm{t}}$-IsoP-M and $\mathrm{Zn}$ appeared somewhat stronger for combined intake of $\mathrm{Zn}$ from both dietary and supplemental sources, compared with associations with dietary $\mathrm{Zn}$ intake alone. Yet, some trials have suggested that $\mathrm{Zn}$ supplementation has little effect on markers of lipid peroxidation, such as $\mathrm{F}_{2}$-IsoP ${ }^{(41,42)}$. Human studies remain scarce, particularly in healthy populations, and further investigation is needed to understand the role of $\mathrm{Zn}$ in oxidative stress reduction.

In our sample, a higher intake of dietary long-chain $n-3$ fatty acids, which has been associated with a lower risk of cardiovascular events ${ }^{(43)}$, was significantly predictive of lower $15-\mathrm{F}_{2 \mathrm{t}}-\mathrm{IsoP}-\mathrm{M}$ and marginally predictive of lower $\mathrm{F}_{2}$-IsoP. 
Supplementation with EPA and DHA, two long-chain $n$ - 3 fatty acids found in fatty fish, has led to decreases in $\mathrm{F}_{2}$-IsoP in some trials $^{(21,44-46)}$ and has had no effect in others ${ }^{(47,48)}$, contrary to previous concerns that higher overall intake of unsaturated fatty acids would increase lipid peroxidation ${ }^{(21)}$. Mas et al. $^{(21)}$ suggest that the reduction in $\mathrm{F}_{2}$-IsoP is related to the antiinflammatory effects of $n-3$ fatty acids. Arachidonic acid, from which $\mathrm{F}_{2}$-isoprostanes are derived, is a primary component of inflammatory cell membranes, but may be partially replaced in membranes by EPA, thereby leading to decreased production of arachidonic acid-derived products.

We observed strong positive associations between trans fat intake and both $\mathrm{F}_{2}$-IsoP and $15-\mathrm{F}_{2 \mathrm{t}}-\mathrm{IsOP}-\mathrm{M}$ concentrations. Trans fats are PUFA, which occur naturally in ruminant fats, but are also formed during the hydrogenation of vegetable oils in industrial processes ${ }^{(49)}$. Some trials have found higher urinary $\mathrm{F}_{2}$-IsoP among participants given trans-fatty acid supplementation $^{(49,50)}$, and it has been suggested that an increase in lipid peroxidation may partially account for the relationship between trans fat and CHD risk ${ }^{(50)}$. Among women enrolled in the SWAN study, Tomey et al. ${ }^{(13)}$ reported an increase in urinary $\mathrm{F}_{2}$-IsoP with higher trans fat intake. However, while their results also suggested positive associations with total fat, SFA, linoleic acid (a PUFA) and oleic acid (a MUFA), we observed no consistent relationships between urinary $\mathrm{F}_{2}$-IsoP and the majority of dietary fat subgroups that we evaluated. Although human investigations remain limited, some evidence from intervention studies has also suggested that $\mathrm{F}_{2}$-IsoP excretion may not be strongly affected by the overall fat content of the diet ${ }^{(51-54)}$. Future studies are needed to evaluate associations between specific dietary fat subgroups and oxidative stress as assessed by $\mathrm{F}_{2}$-IsoP.

With adjustments for confounders such as age, BMI and daily intake of fruits and vegetables, total physical activity was inversely associated with $\mathrm{F}_{2}$-IsoP, with a similar but nonsignificant trend for $15-\mathrm{F}_{2 \mathrm{t}}$-IsoP-M. To avoid problems of collinearity, we chose not to control for multiple related dietary factors in the same model. Thus, adjustment only for fruits and vegetables may be insufficient to account for confounding by diet, given strong correlations between most dietary intakes and total physical activity in our sample. Although our findings are consistent with several aerobic exercise trials among women $^{(55-58)}$, our interest was in the combination of activity from both exercise and daily activities, and thus the results may not be directly comparable. Habitual physical activity is thought to potentially decrease oxidative stress through adaptive processes, in which levels of antioxidant enzymes and water- and lipid-soluble antioxidants may increase ${ }^{(59)}$. However, observational studies of habitual physical activity and $\mathrm{F}_{2}$-isoprostanes among premenopausal women have been conflicting ${ }^{(60,61)}$, and further investigation is warranted.

The evaluation of $15-\mathrm{F}_{2 \mathrm{t}}$-IsoP-M, a biomarker used in a few previous studies, is a unique strength of this study. In addition, owing to extensive baseline data collection in the Sister Study, we were able to control for the major factors known to affect oxidative stress. However, there are some limitations including the reliance on self-reported measures of diet, alcohol consumption and physical activity. Measurement error is inherent to the FFQ, and under- or over-reporting of physical activity and alcohol consumption may be a concern. Although more objective measures may be preferable, they would likely be infeasible in a sample as large as ours. Furthermore, the CV for the assays of $\mathrm{F}_{2}$-IsoP and $15-\mathrm{F}_{2 \mathrm{t}}$-IsoP-M were somewhat high, suggesting caution in the interpretation of our results. Participants in this study were largely homogeneous with respect to demographic characteristics, limiting our ability to generalise to males or non-white populations. Given the large number of associations that we evaluated, there is also a risk of falsepositive results. However, all tests were based on a priori hypotheses. Finally, we were unable to address differences in $\mathrm{F}_{2}$-IsoP concentrations by intensity of physical activity or type of alcohol consumption. The effects of long-term, moderateintensity physical activity on oxidative stress may differ from those of acute, vigorous activity ${ }^{(23,24)}$, whereas the effects of red wine may differ from those of other alcoholic beverages due to its antioxidant content ${ }^{(62,63)}$. Evaluation of such associations could further our understanding of the influence of lifestyle factors on oxidative stress.

In summary, the results of this study suggest that physical activity and specific dietary factors, such as antioxidant nutrients and long-chain $n-3$ fatty acids, may be inversely associated with oxidative stress among premenopausal women. Our findings also suggest that higher intake of trans fats may be associated with higher levels of oxidative stress. Future studies are warranted to evaluate additional biomarkers of oxidative stress in more diverse populations.

\section{Acknowledgements}

The authors appreciate the helpful comments of Dr Kelly Ferguson and Dr Yong-Moon Park.

This research was supported in part by the Intramural Research Program of the National Institutes of Health, National Institute of Environmental Health Sciences (Z01-ES044005), the Avon Foundation (02-2012-085) and by the National Center for Advancing Translational Sciences (KL2-TR001109).

C. A., H. B. N., D. P. S. designed the study; D. P. S., G. L. M. conducted the study; C. A., H. B. N. analysed the data; all the authors contributed to writing of the paper and had primary responsibility for the final content. All the authors read and approved the final version of the manuscript.

The authors declare that there are no conflicts of interest.

\section{Supplementary material}

For supplementary material/s referred to in this article, please visit http://dx.doi.org/doi:10.1017/S0007114516003226

\section{References}

1. Zhang ZJ (2013) Systematic review on the association between F2-isoprostanes and cardiovascular disease. Ann Clin Biochem 50, 108-114.

2. Epplein M, Franke AA, Cooney RV, et al. (2009) Association of plasma micronutrient levels and urinary isoprostane with risk of lung cancer: the Multiethnic Cohort Study. Cancer Epidemiol Biomarkers Prev 18, 1962-1970. 
3. Dai Q, Gao YT, Shu XO, et al. (2009) Oxidative stress, obesity, and breast cancer risk: results from the Shanghai Women's Health Study. J Clin Oncol 27, 2482-2488.

4. Maiese K (2015) New insights for oxidative stress and diabetes mellitus. Oxid Med Cell Longev 2015, 875961

5. Barocas DA, Motley S, Cookson MS, et al. (2011) Oxidative stress measured by urine F2-isoprostane level is associated with prostate cancer. J Urol 185, 2102-2107.

6. Morrow JD, Frei B, Longmire AW, et al. (1995) Increase in circulating products of lipid peroxidation (F2-isoprostanes) in smokers. Smoking as a cause of oxidative damage. $N$ Engl $J$ Med 332, 1198-1203.

7. Aseervatham GS, Sivasudha T, Jeyadevi R, et al. (2013) Environmental factors and unhealthy lifestyle influence oxidative stress in humans - an overview. Environ Sci Pollut Res Int 20, 4356-4369.

8. Mayne ST (2003) Antioxidant nutrients and chronic disease: use of biomarkers of exposure and oxidative stress status in epidemiologic research. J Nutr 133, Suppl. 3, 933S-940S.

9. Di Mascio P, Murphy ME \& Sies H (1991) Antioxidant defense systems: the role of carotenoids, tocopherols, and thiols. Am J Clin Nutr 53, 194s-200s.

10. Sies H, Stahl W \& Sundquist AR (1992) Antioxidant functions of vitamins. Vitamins $\mathrm{E}$ and $\mathrm{C}$, beta-carotene, and other carotenoids. Ann N Y Acad Sci 669, 7-20.

11. Cruz KJ, de Oliveira AR \& Marreiro Ddo N (2015) Antioxidant role of zinc in diabetes mellitus. World J Diabetes 6, 333-337.

12. Dorjgochoo T, Gao YT, Chow WH, et al. (2012) Major metabolite of $\mathrm{F} 2$-isoprostane in urine may be a more sensitive biomarker of oxidative stress than isoprostane itself. Am J Clin Nutr 96, 405-414.

13. Tomey KM, Sowers MR, Li X, et al. (2007) Dietary fat subgroups, zinc, and vegetable components are related to urine F2a-isoprostane concentration, a measure of oxidative stress, in midlife women. J Nutr 137, 2412-2419.

14. Cocate PG, Natali AJ, Alfenas RC, et al. (2015) Carotenoid consumption is related to lower lipid oxidation and DNA damage in middle-aged men. Br J Nutr 114, 257-264.

15. Holt EM, Steffen LM, Moran A, et al. (2009) Fruit and vegetable consumption and its relation to markers of inflammation and oxidative stress in adolescents. J Am Diet Assoc 109, 414-421.

16. Helmersson J, Arnlov J, Larsson A, et al. (2009) Low dietary intake of beta-carotene, alpha-tocopherol and ascorbic acid is associated with increased inflammatory and oxidative stress status in a Swedish cohort. Br J Nutr 101, 1775-1782.

17. Thomson CA, Stendell-Hollis NR, Rock CL, et al. (2007) Plasma and dietary carotenoids are associated with reduced oxidative stress in women previously treated for breast cancer. Cancer Epidemiol Biomarkers Prev 16, 2008-2015.

18. Rossner P Jr, Gammon MD, Terry MB, et al. (2006) Relationship between urinary 15-F2t-isoprostane and 8-oxodeoxyguanosine levels and breast cancer risk. Cancer Epidemiol Biomarkers Prev 15, 639-644.

19. Rink SM, Mendola P, Mumford SL, et al. (2013) Self-report of fruit and vegetable intake that meets the 5 a day recommendation is associated with reduced levels of oxidative stress biomarkers and increased levels of antioxidant defense in premenopausal women. $J$ Acad Nutr Diet 113, 776-785.

20. Root MM, McGinn MC, Nieman DC, et al. (2012) Combined fruit and vegetable intake is correlated with improved inflammatory and oxidant status from a cross-sectional study in a community setting. Nutrients $\mathbf{4}, 29-41$.

21. Mas E, Woodman RJ, Burke V, et al. (2010) The omega-3 fatty acids EPA and DHA decrease plasma F(2)-isoprostanes: results from two placebo-controlled interventions. Free Radic Res 44, 983-990.
22. Kiecolt-Glaser JK, Epel ES, Belury MA, et al. (2013) Omega-3 fatty acids, oxidative stress, and leukocyte telomere length: a randomized controlled trial. Brain Behav Immun 28, 16-24.

23. Mastaloudis A, Leonard SW \& Traber MG (2001) Oxidative stress in athletes during extreme endurance exercise. Free Radic Biol Med 31, 911-922.

24. Nikolaidis MG, Kyparos A \& Vrabas IS (2011) F(2)-isoprostane formation, measurement and interpretation: the role of exercise. Prog Lipid Res 50, 89-103.

25. Das SK \& Vasudevan DM (2007) Alcohol-induced oxidative stress. Life Sci 81, 177-187.

26. Montuschi P, Barnes PJ \& Roberts LJ 2nd (2004) Isoprostanes: markers and mediators of oxidative stress. FASEB $J \mathbf{1 8}$, $1791-1800$

27. Milne GL, Dai Q \& Roberts LJ 2nd (2015) The isoprostanes 25 years later. Biochim Biophys Acta 1851, 433-445.

28. Dorjgochoo T, Gao YT, Chow WH, et al. (2011) Obesity, age, and oxidative stress in middle-aged and older women. Antioxid Redox Signal 14, 2453-2460.

29. Il'yasova D, Scarbrough P \& Spasojevic I (2012) Urinary biomarkers of oxidative status. Clin Chim Acta 413, 1446-1453.

30. Morrow JD, Zackert WE, Yang JP, et al. (1999) Quantification of the major urinary metabolite of 15-F2t-isoprostane (8-iso-PGF2alpha) by a stable isotope dilution mass spectrometric assay. Anal Biochem 269, 326-331.

31. Basu S \& Helmersson J (2005) Factors regulating isoprostane formation in vivo. Antioxid Redox Signal 7, 221-235.

32. Milne GL, Sanchez SC, Musiek ES, et al. (2007) Quantification of F2-isoprostanes as a biomarker of oxidative stress. Nat Protoc 2, 221-226.

33. Morrow JD \& Roberts LJ 2nd (1999) Mass spectrometric quantification of F2-isoprostanes in biological fluids and tissues as measure of oxidant stress. Methods Enzymol 300, 3-12.

34. Morales CR, Terry ES, Zackert WE, et al. (2001) Improved assay for the quantification of the major urinary metabolite of the isoprostane $15-\mathrm{F}(2 \mathrm{t})$-Isoprostane (8-iso-PGF(2alpha)) by a stable isotope dilution mass spectrometric assay. Clin Chim Acta 314, 93-99.

35. Il'yasova D, Morrow JD, Ivanova A, et al. (2004) Epidemiological marker for oxidant status: comparison of the ELISA and the gas chromatography/mass spectrometry assay for urine 2,3-dinor5,6-dihydro-15-F2t-isoprostane. Ann Epidemiol 14, 793-797.

36. Block G, Hartman AM, Dresser CM, et al. (1986) A data-based approach to diet questionnaire design and testing. $\mathrm{Am} \mathrm{J}$ Epidemiol 124, 453-469.

37. Basiotis PP CA, Gerrior SA, Juan WY, et al. (2002). The Healthy Eating Index: 1999-2000. Washington, DC: United States Department of Agriculture, Center for Nutrition Policy and Promotion.

38. Ainsworth BE, Haskell WL, Whitt MC, et al. (2000) Compendium of physical activities: an update of activity codes and MET intensities. Med Sci Sports Exerc 32, S498-S504.

39. Young AJ \& Lowe GM (2001) Antioxidant and prooxidant properties of carotenoids. Arch Biochem Biophys 385, 20-27.

40. Prasad AS (2014) Zinc: an antioxidant and anti-inflammatory agent: role of zinc in degenerative disorders of aging. $J$ Trace Elem Med Biol 28, 364-371.

41. Andriollo-Sanchez M, Hininger-Favier I, Meunier $\mathrm{N}$, et al. (2008) No antioxidant beneficial effect of zinc supplementation on oxidative stress markers and antioxidant defenses in middle-aged and elderly subjects: the Zenith study. J Am Coll Nutr 27, 463-469.

42. Seet RC, Lee CY, Lim EC, et al. (2011) Oral zinc supplementation does not improve oxidative stress or vascular function in patients with type 2 diabetes with normal zinc levels. Atherosclerosis 219, 231-239. 
43. Delgado-Lista J, Perez-Martinez $\mathrm{P}$, Lopez-Miranda J, et al. (2012) Long chain omega-3 fatty acids and cardiovascular disease: a systematic review. Br J Nutr 107, Suppl. 2, S201-S213.

44. Mori TA, Woodman RJ, Burke V, et al. (2003) Effect of eicosapentaenoic acid and docosahexaenoic acid on oxidative stress and inflammatory markers in treated-hypertensive type 2 diabetic subjects. Free Radic Biol Med 35, 772-781.

45. Nalsen C, Vessby B, Berglund L, et al. (2006) Dietary (n-3) fatty acids reduce plasma $\mathrm{F} 2$-isoprostanes but not prostaglandin F2alpha in healthy humans. J Nutr 136, 1222-1228.

46. Skarke C, Alamuddin N, Lawson JA, et al. (2015) Bioactive products formed in humans from fish oils. J Lipid Res $\mathbf{5 6}$, 1808-1820.

47. Kirkhus B, Lamglait A, Eilertsen KE, et al. (2012) Effects of similar intakes of marine $n$-3 fatty acids from enriched food products and fish oil on cardiovascular risk markers in healthy human subjects. Br J Nutr 107, 1339-1349.

48. Wu WH, Lu SC, Wang TF, et al. (2006) Effects of docosahexaenoic acid supplementation on blood lipids, estrogen metabolism, and in vivo oxidative stress in postmenopausal vegetarian women. Eur J Clin Nutr 60, 386-392.

49. Kuhnt K, Wagner A, Kraft J, et al. (2006) Dietary supplementation with 11trans- and 12trans-18:1 and oxidative stress in humans. Am J Clin Nutr 84, 981-988.

50. Smit LA, Katan MB, Wanders AJ, et al. (2011) A high intake of trans fatty acids has little effect on markers of inflammation and oxidative stress in humans. J Nutr 141, 1673-1678.

51. Marina A, von Frankenberg AD, Suvag S, et al. (2014) Effects of dietary fat and saturated fat content on liver fat and markers of oxidative stress in overweight/obese men and women under weight-stable conditions. Nutrients 6, 4678-4690.

52. Richelle M, Turini ME, Guidoux R, et al. (1999) Urinary isoprostane excretion is not confounded by the lipid content of the diet. FEBS Lett 459, 259-262.

53. Chen G, Heilbrun LK, Venkatramanamoorthy R, et al. (2004) Effects of low-fat and/or high-fruit-and-vegetable diets on plasma levels of 8-isoprostane-F2alpha in the Nutrition and Breast Health study. Nutr Cancer 50, 155-160.

54. Egert S, Kratz M, Kannenberg F, et al. (2011) Effects of high-fat and low-fat diets rich in monounsaturated fatty acids on serum lipids, LDL size and indices of lipid peroxidation in healthy non-obese men and women when consumed under controlled conditions. Eur J Nutr 50, 71-79.

55. Devries MC, Hamadeh MJ, Glover AW, et al. (2008) Endurance training without weight loss lowers systemic, but not muscle, oxidative stress with no effect on inflammation in lean and obese women. Free Radic Biol Med 45, 503-511.

56. Schmitz KH, Warren M, Rundle AG, et al. (2008) Exercise effect on oxidative stress is independent of change in estrogen metabolism. Cancer Epidemiol Biomarkers Prev 17, 220-223.

57. Karolkiewicz J, Michalak E, Pospieszna B, et al. (2009) Response of oxidative stress markers and antioxidant parameters to an 8-week aerobic physical activity program in healthy, postmenopausal women. Arch Gerontol Geriatr 49 , e67-e71.

58. Campbell PT, Gross MD, Potter JD, et al. (2010) Effect of exercise on oxidative stress: a 12-month randomized, controlled trial. Med Sci Sports Exerc 42, 1448-1453.

59. Aldred S (2007) Oxidative and nitrative changes seen in lipoproteins following exercise. Atherosclerosis 192, 1-8.

60. Rudra CB, Wactawski-Wende J, Hovey KM, et al. (2011) Energy expenditure and plasma F2-isoprostanes across the menstrual cycle. Med Sci Sports Exerc 43, 785-792.

61. Sowers M, McConnell D, Jannausch ML, et al. (2008) Oestrogen metabolites in relation to isoprostanes as a measure of oxidative stress. Clin Endocrinol (Oxf) 68, 806-813.

62. Das DK, Sato M, Ray PS, et al. (1999) Cardioprotection of red wine: role of polyphenolic antioxidants. Drugs Exp Clin Res 25, 115-120.

63. Covas MI, Gambert P, Fito M, et al. (2010) Wine and oxidative stress: up-to-date evidence of the effects of moderate wine consumption on oxidative damage in humans. Atherosclerosis 208, 297-304. 